\title{
First data on antimicrobial susceptibility patterns of Moraxella catarrhalis isolates in Lebanon
}

Monzer Hamze ${ }^{1}$, Marwan Osman', Hassan Mallat ${ }^{1}$, Marcel Al Achkar²

\section{Abstract}

Background: Moraxella catarrhalis is an important bacterial pathogen. However, no data regarding this human pathogenare currently available in Lebanon. This study aimed to determine for the first time the antimicrobial susceptibility profiles of $M$. catarrhalis isolates in Lebanon.

Methods: A total of $34 \mathrm{M}$. catarrhalis strains were isolated from clinical specimensduring the period from November 2010 to March 2019. Bacterial identification was performed using MALDI-TOF MS. Antibiotic susceptibility of allisolates was interpreted according to EUCAST recommendations.

Results: A total of 34 non-duplicated $M$. catarrhalis strains were isolated from patients referred to Nini Hospital in Tripoli, Lebanon. Regarding antibiotic susceptibility rates, the percent susceptibility is $100 \%$ to the majority of antibiotics, except ampicillin (7.4\%), trimethoprimsulfamethoxazole (85.3\%), nalidixic acid (85.3\%), and ciprofloxacin (97.1\%).

Conclusion: To our knowledge, this study is the first investigation regarding the antimicrobial susceptibility patterns of $M$. catarrhalis isolates in Lebanon. In addition to the high level of resistance to ampicillin, our findings showed the emergence of resistance to trimethoprimsulfamethoxazole, nalidixic acid and ciprofloxacin. Even if this study provides useful information to develop effective empirical treatment, we recommend the implementation of reliable diagnostic tools to guide appropria tetreatment.

1 Laboratoire Microbiologie, Santé et Environnement (LMSE), Doctoral School of Sciences and Technology, Faculty of Public Health, Lebanese University, Tripoli, Lebanon.

2 Clinical Laboratory, Nini Hospital, Tripoli, Lebanon.

Contact information:

Prof. Monzer Hamze.

$\equiv$ mhamze@monzerhamze.com 


\section{Introduction}

Moraxella catarrhalis is an aerobic Gram-negative diplococcus, formerly known as Branhamella catarrhalis, that resides exclusively in humans, and commensally colonizes the mucosal surface of the upper respiratory tract, and occasionally the conjunctiva and genital tract [1]. The highest prevalence of colonization was detected among infants and children, which decreased in healthy adults [2]. The role of this bacterium as a disease-causing organism has long been questioned. M. catarrhalis was recognized to cause occasionally infections such as acute otitis media, sinusitis, acute bronchitis, pneumonia, and exacerbations of chronic obstructive pulmonary disease, and rarely bacteremia, meningitis, septic arthritis, osteomyelitis, endocarditis, and pericarditis, especially in immunocompromised persons [3]. In fact, the absence of vaccines for prevention and the low number of active antibiotics for treatment of $M$. catarrhalis infections, have considered this bacterium as an important human pathogen [4]. In addition, two major resistance mechanisms have been described in $M$. catarrhalis: the inactivation of antimicrobials by enzymes such as $\beta$-lactamases and the decrease in permeability of bacterial cell wall (reducing in the number of porins and/or enhancement of the active efflux system) [1]. The beta-lactamase-producing $M$. catarrhalis was firstly reported in 1976. Today, two distinct BRO-type $\beta$-lactamase enzymes (BRO-1 and BRO-2), have been reported worldwide [5]. Regrettably, BRO-positive $M$. catarrhalis strains have increased rapidly in recent years, and are now accounting for more than $90 \%$ globally [2]. The evidence of continuing misuse of antibiotics associated with a low level of antibiotic awareness is of global concern [6]. In Lebanon, as other developing countries, national investigations showed an increase in the levels of antimicrobial resistance in clinical and non-clinical settings [7-11]. For instance, a recent nationwide study conducted in 13 different hospitals located in different Lebanese governorates showed a trend of increasing antimicrobial resistan- ce [12]. Even if the epidemiology of antimicrobial resistance is widely studied in Lebanon, there is a lack of studies on antimicrobial resistance in $\mathrm{M}$. catarrhalis isolates in this country. Therefore, we decided to assess for the first time the antimicrobial resistance patterns in $M$. catarrhalis strains isolated in North Lebanon.

\section{Material and Methods}

This study was conducted in the clinical microbiology laboratory of Nini Hospital during the period from November 2010 to March 2019. The isolation of $M$. catarrhalis strains was performed according to standard protocols proposed by the Référentielen Microbiologie Médicale (REMIC) using a calibrated loop $(10 \mu \mathrm{l})$ and a blood agar (Bio-Rad ${ }^{\circledR}$, France) after an incubation for 18 to 24 hours at $35^{\circ} \mathrm{C}$ in air enriched with $5 \% \mathrm{CO}_{2}$. Bacterial identification was carriedout through the use of API-NH (bioMérieux, Marcy l'Etoile, France). All isolates were transferred to the Laboratoire Microbiologie Santé et Environnement (LMSE) at the Lebanese University for identification confirmation or exclusion using matrix assisted laser desorption ionization-time of flight mass spectrometry (MALDI-TOF-MS) (bioMérieux, Marcy l'Etoile, France). The antibiotic susceptibility testing was performed for $M$. catarrhalis isolates by the disk diffusion method on Mueller Hinton supplemented with $5 \%$ blood and $20 \mathrm{mg} / \mathrm{l} \beta-\mathrm{NAD}$ ( $\mathrm{MH}-\mathrm{F}$ ) according to the recommendations of the European Committee on Antimicrobial Susceptibility Testing (EUCAST). The antibiotics tested were ampicillin (AMP; $2 \mu \mathrm{g}$ ), amoxicillin - clavulanic acid (AMC; 2-1 $\mu \mathrm{g})$, cefotaxime (CTX; $5 \mu \mathrm{g})$, cefixime (CFM; 5 $\mu \mathrm{g})$, tetracycline (TET; $30 \mu \mathrm{g})$, minocycline (MNO; 30 $\mu \mathrm{g})$, erythromycin $(\mathrm{E} ; 15 \mu \mathrm{g})$, chloramphenicol (C; 30 $\mu \mathrm{g})$, nalidixic acid (NA; $30 \mu \mathrm{g})$, ciprofloxacin (CIP; 5 $\mu \mathrm{g})$ and trimethoprim-sulfamethoxazole (SXT; 1.25 $23.75 \mu \mathrm{g})$. 


\section{Results}

This study was conducted in the North governorate of Lebanon. A total of 34 non-duplicated $M$. catarrhalis isolates were recovered from patients referred to Nini Hospital. Overall, M. catarrhalis strains were isolated from nose $(n=19)$, ear $(n=7)$, sputum $(n=5)$, blood $(n=1)$, eye $(n=1)$, and throat $(n=1)$ of 34 patients (23 malesand 11 females, ranging in age from 2 weeks to 77 years, with a mean age of 11.9 years) presenting respiratory like symptoms. Regarding antibiotic susceptibility rates, the percentsusceptibility is $100 \%$ to the majority of antibiotics, such as amoxicillin-clavulanic acid, cefotaxime, cefixime,tetracycline, minocycline, erythromycin, and chloramphenicol (Table 1). Higher antibioticresistance rates were observed to ampicillin (92.6\%), trimethoprim-sulfamethoxazole (14.7\%), nalidixic acid (14.7\%), and ciprofloxacin (2.9\%) (Figure 1).

Figure 1: Antibiotic susceptibility rates of MoraxeIla catarrhalis isolates.

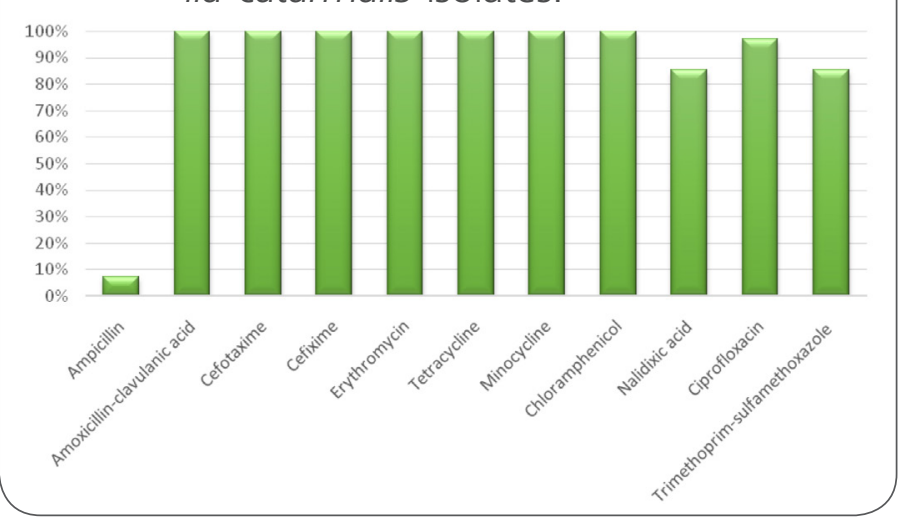

\section{Discussion}

The global increase in the prevalence of $M$. catarrhalis in recent years has drawn the attention to the clinical importance of this bacterium, particularly as an important cause of respiratory tract infections [13]. The present surveillance study in North Lebanon aimed to evaluate the susceptibility patterns of M. catarrhalis clinical isolates to several commonly used antibiotic agents in the hopes of helping physicians and infectious diseases specialists in developing effective empirical antibiotic treatment for M. catarrhalis infections. These bacteria are almost universally capable of producing of one of two chromosomally encoded BRO $\beta$-lactamases, with previous investigations showing production in more than $90 \%$ of clinical isolates [2, 14-16]. As expected, the present finding (92.6\% of isolates are ampicillin resistant) is compatible with previous investigations (Table 2). This dramatic increase in the prevalence of ampicillin resistant $M$. catarrhalis strains could be regarded as the fastest dissemination of $\beta$-lactamase genes within the genus. The BRO genes appear to be chromosomally located but are readily transferred by conjugation with in the Moraxella species. Besides the high level of resistance to penicillin, the combination of penicillin with a $\beta$-lactamase inhibitor used as a treatment of $M$. catarrhalis infections is still active against $100 \%$ of our isolates in almost all countries. However, unfortunately, recent studies found the emergence of $M$. catarrhalis strains resis-

Table 1. Distribution of Moraxella catarrhalis isolates according to clinical specimens, and their antibiotic resistance patterns.

\begin{tabular}{|l|c|c|c|c|c|c|c|c|c|c|c|c|}
\hline \multicolumn{10}{c|}{ Antibiotic agents (\% of resistance) } \\
\hline Specimen & N & AMP & AMC & CTX & CFM & E & TET & MNO & C & NA & CIP & SXT \\
\hline Nose & 19 & 84.2 & 0 & 0 & 0 & 0 & 0 & 0 & 0 & 15.8 & 0 & 15.8 \\
\hline Ear & 7 & 100 & 0 & 0 & 0 & 0 & 0 & 0 & 0 & 14.3 & 0 & 28.6 \\
\hline Sputum & 5 & 100 & 0 & 0 & 0 & 0 & 0 & 0 & 0 & 20 & 20 & 0 \\
Blood & 1 & 100 & 0 & 0 & 0 & 0 & 0 & 0 & 0 & 0 & 0 & 0 \\
Eye & 1 & 100 & 0 & 0 & 0 & 0 & 0 & 0 & 0 & 0 & 0 & 0 \\
\hline Throat & 1 & 100 & 0 & 0 & 0 & 0 & 0 & 0 & 0 & 0 & 0 & 0
\end{tabular}


Table 2. Worldwide available data regarding the percentage of resistance of Moraxella catarrhalis isolates to major antibiotics.

\begin{tabular}{|l|c|c|c|c|c|c|c|c|c|}
\hline \multicolumn{1}{|c|}{} & \multicolumn{8}{c|}{ Antibiotic agents } \\
\hline \multicolumn{1}{|c|}{ Country } & Year & AMP & AMC & CTX & E & TET & CIP & SXT & Ref. \\
\hline Australia & 2010 & 63.6 & 0 & 0 & 0 & 1.1 & 0 & 13.8 & 22 \\
\hline United States & 2012 & 96.4 & 0 & 0.2 & 0.5 & 0.2 & 0 & 2.5 & 33 \\
\hline United States & 2009 & 95.2 & 4.8 & 3.2 & ND & ND & ND & ND & 17 \\
\hline Canada & 2014 & ND & 0 & 0 & ND & 0 & 0 & 0 & 18 \\
\hline Canada & 2000 & ND & 0 & 0 & ND & 0.7 & 0 & 15.7 & 23 \\
\hline Portugal & 2001 & 81.6 & 0 & 0 & ND & 0 & 0 & ND & 34 \\
\hline Greece & 2014 & 47.8 & 0 & 0 & ND & 1.5 & 0 & 28.4 & 24 \\
\hline Ethiopia & 2018 & 94.6 & ND & ND & 86.6 & 37.8 & 16.2 & 78.4 & 20 \\
\hline Tunisia & 2008 & 95 & 0 & 0 & 3.8 & 1.25 & 0 & 12.5 & 21 \\
\hline Iran & 2012 & 0 & 0 & 0 & 0 & ND & 0 & 100 & 25 \\
\hline Taiwan & 2012 & 97.8 & 0 & 0 & ND & 19.8 & 0 & 18.5 & 2 \\
\hline China & 2018 & 74.2 & 0 & 0 & 70.8 & 6.8 & 3.4 & 28.1 & 19 \\
\hline Thailand & 2016 & 97 & 0 & 0 & ND & ND & ND & ND & 16 \\
\hline Pakistan & 2015 & 63 & 4 & 0 & 59.1 & ND & 59 & 59 & 13 \\
\hline Lebanon & 2019 & 92.6 & 0 & 0 & 0 & 0 & 2.9 & 14.7 & This study \\
\hline & Ampicillin (AMP); Amoxicillin - clavulanic acid (AMC); Cefotaxime (CTX); Erythromycin (E); Tetracycline (TET); & Ciprofloxacin (CIP); Trimethoprim-sulfamethoxazole (SXT); Not determined (ND).
\end{tabular}

tant to amoxicillin-clavulanic acid and third generation cephalosporins [17].

Other antimicrobial agents, such as tetracycline, erythromycin, ciprofloxacin, and trimethoprim-sulfamethoxazole have also been empirically used to treat $M$. catarrhalis infections worldwide with apparent success. All clinical isolates collected in this study showed $100 \%$ susceptibility to tetracycline and erythromycin which is consistent with the most current international research [18]. Nevertheless, the antimicrobial resistance threat must keep the clinical community vigilant. Regrettably, tetracycline resistance has emerged in numerous developing and developed countries. The higher levels of resistance were reported in China [19], Taiwan [2] and Ethiopia [20]. Furthermore, several studies realized in developing countries indicated unexpected levels of antimicrobial resistance to erythromycin in $M$. ca- tarrhalis isolates, with $59.1 \%$ in Pakistan [13], 70.8\% in China [19], and 86.6\% in Ethiopia [20].

On the other hand, our findings showed the emergence of resistance to trimethoprim-sulfamethoxazole (14.7\%), and ciprofloxacin (2.9\%). Various previous studies conducted globally reported the spread of resistance to trime thoprim-sulfamethoxazole among $M$. catarrhalis isolates, such as $12.5 \%$ in Tunisia [21], $13.8 \%$ in Australia [22], $15.7 \%$ in Canada [23], $18.5 \%$ in Taiwan [2], 28.1\% in China [19], 28.4\% in Greece [24], 59\% in Pakistan [13], 78.4\% in Ethiopia [20], and 100\% in Iran [25]. Based on the aforementioned data, the spread of cotrimoxazole resistance among $M$. catarrhalis isolates in developing countries is shocking, and reveals a prominent threat jeopardizing the clinical efficacy of this important antibiotic. Moreover, the ciprofloxacin resistancewas detected for the first time among 
M. catarrhalis isolates in Lebanon, with a higher rate than that reported in the majority of countries. Surprisingly, two studies conducted in Ethiopia and Pakistan showed high level of resistance to ciprofloxacin, with $16.2 \%$ and $59 \%$ respectively $[13,20]$.

Indeed, our generated data are in accordance with recent national studies reporting an increased level of antimicrobial resistance among infectious bacterial agents, a striking low level of antibiotic awareness among Lebanese population, a spread of counterfeit medicines, and a misuse of antibiotics in Lebanon [6-11, 26-32]. Even if this study provides useful information for physicians to develop effective empirical antibiotic treatment for $M$. catarrhalis infections, we advise to pay attention to this prominent issue and we recommend the implementation of reliable identification and antibiotic susceptibility testing in routine laboratory diagnostics to guide appropriate treatment.

In summary, to our knowledge, this study is the first investigation regarding the antimicrobial susceptibility patterns of $M$. catarrhalis isolates in Lebanon. Overall, our isolates showed $100 \%$ susceptibility to the majority of tested antibiotics, except ampicillin, trimethoprim-sulfamethoxazole, nalidixic acid and ciprofloxacin. Due to the limited number of isolates analyzed in this investigations, the epidemiologic significance of these results remains to be confirmed. Therefore, additional long-term surveillance studies including a large number of isolates are required to assess the antimicrobial resistance of this important human pathogen.

\section{References}

1. Spaniol V, Bernhard S, Aebi C. Moraxella catarrhalis AcrABOprM efflux pump contributes to antimicrobial resistance and is enhanced during cold shock response. Antimicrob Agents Chemother. 2015; 59(4):1886-94.

2. Hsu SF, Lin YT, Chen TL, Siu LK, Hsueh PR, Huang ST, et al. Antimicrobial resistance of Moraxella catarrhalis isolates in Taiwan. J Microbiol Immunol Infect. 2012; 45(2):134-40.

3. Aebi C. Moraxella catarrhalis - pathogen or commensal? Adv Exp Med Biol. 2011; 697:107-16.
4. Gumerova NI, Al-Sayed E, Krivosudsky L, Cipcic-Paljetak H, Verbanac D, Rompel A. Antibacterial Activity of Polyoxometalates Against Moraxella catarrhalis. Front Chem. 2018; 6:336.

5. Khan MA, Northwood JB, Levy F, Verhaegh SJ, Farrell DJ, Van Belkum A, et al. bro \{beta\}-lactamase and antibiotic resistances in a global cross-sectional study of Moraxella catarrhalis from children and adults. J Antimicrob Chemother. 2010; 65(1):91-7.

6. Al Omari S, Al Mir H, Wrayde S, Merhabi S, Dhaybi I, Jamal $S$, et al. First Lebanese Antibiotic Awareness Week campaign: knowledge, attitudes and practices towards antibiotics. J Hosp Infect. 2019; 101(4):475-9.

7. Jamal S, Al Atrouni A, Rafei R, Dabboussi F, Hamze M, Osman M. Molecular mechanisms of antimicrobial resistance in Acinetobacter baumannii, with a special focus on its epidemiology in Lebanon. J Glob Antimicrob Resist. 2018; 15:154-63.

8. El Moujaber G, Osman M, Rafei R, Dabboussi F, Hamze M. Molecular mechanisms and epidemiology of resistance in Streptococcus pneumoniae in the Middle East region. J Med Microbiol. 2017; 66(7):847-58.

9. Osman M, Al Mir H, Rafei R, Dabboussi F, Madec JY, Haenni M, et al. Epidemiology of Antibiotic Resistance in Lebanese ExtraHospital Settings: an overview. J Glob Antimicrob Resist. 2018; 17:123-129

10. Hamze M, Osman M, Mallat H, Nasr S, BouRaad E, Achkar M. Epidemiology and antibiotic susceptibility patterns of carbapenem-resistant Gram-negative bacteria isolated from two tertiary care hospitals in North Lebanon. Int Arab J Antimicrob Agents. 2018; 8(2:3).

11. Osman M, Mallat $H$, Hamze M, Achkar M. Prevalence and antibiotic susceptibility patterns of bacteria causing urinary tract infections in Youssef Hospital Center: first report from Akkar governorate, North Lebanon. Int Arab J Antimicrob Agents. 2017; 7(1:2).

12. Moghnieh R, Araj GF, Awad L, Daoud Z, Mokhbat JE, Jisr T, et al. A compilation of antimicrobial susceptibility data from a network of 13 Lebanese hospitals reflecting the national situation during 2015-2016. Antimicrob Resist Infect Control. 2019; 8:41.

13. Shaikh SB, Ahmed Z, Arsalan SA, Shafiq S. Prevalence and resistance pattern of Moraxella catarrhalis in communityacquired lower respiratory tract infections. Infect Drug Resist. 2015; 8:263-7.

14. Murphy TF, Parameswaran GI. Moraxella catarrhalis, a human respiratory tract pathogen. Clin Infect Dis. 2009; 49(1):124-31.

15. Doern GV, Jones RN, Pfaller MA, Kugler K. Haemophilus influenzae and Moraxella catarrhalis from patients with community-acquired respiratory tract infections: antimicrobial susceptibility patterns from the SENTRY antimicrobial Surveillance Program (United States and Canada, 1997). Antimicrob Agents Chemother. 1999; 43(2):385-9. 
16. Srifuengfung S, Tribuddharat C, Phoomniyom S, Chuanphung S. Prevalence and antimicrobial susceptibility of Haemophilus influenzae and Moraxella catarrhalis isolated from patients in Bangkok, Thailand. J Glob Antimicrob Resist. 2016; 5:86-7.

17. Harrison CJ, Woods C, Stout G, Martin B, Selvarangan R. Susceptibilities of Haemophilus influenzae, Streptococcus pneumoniae, including serotype 19A, and Moraxella catarrhalis paediatric isolates from 2005 to 2007 to commonly used antibiotics. J Antimicrob Chemother. 2009; 63(3):511-9.

18. Bandet T, Whitehead S, Blondel-Hill E, Wagner K, Cheeptham $\mathrm{N}$. Susceptibility of clinical Moraxella catarrhalis isolates in British Columbia to six empirically prescribed antibiotic agents. Can J Infect Dis Med Microbiol. 2014; 25(3):155-8.

19. Shi W, Wen D, Chen C, Yuan L, Gao W, Tang P, et al. betaLactamase production and antibiotic susceptibility pattern of Moraxella catarrhalis isolates collected from two county hospitals in China. BMC Microbiol. 2018; 18(1):77.

20. Mulu W, Yizengaw E, Alemu M, Mekonnen D, Hailu D, Ketemaw K, et al. Pharyngeal colonization and drug resistance profiles of Morraxella catarrhalis, Streptococcus pneumoniae, Staphylococcus aureus, and Haemophilus influenzae among HIV infected children attending ART Clinic of Felegehiwot Referral Hospital, Ethiopia. PLoS One. 2018; 13(5):e0196722.

21. Khemiri $H$, Smaoui $H$, Kechrid A. [Antimicrobial susceptibility of 80 Moraxella catarrhalis strains isolated in the children's hospital of Tunis]. Pathol Biol (Paris). 2008; 56(3):158-61.

22. Pingault NM, Bowman JM, Lehmann D, Riley TV. Antimicrobial susceptibility of Moraxella catarrhalis isolated from children in Kalgoorlie-Boulder, Western Australia. Pathology. 2010; 42(3):273-9.

23. Zhanel GG, Karlowsky JA, Low DE, Hoban DJ. Antibiotic resistance in respiratory tract isolates of Haemophilus influenzae and Moraxella catarrhalis collected from across Canada in 19971998. J Antimicrob Chemother. 2000; 45(5):655-62.

24. Maraki S, Papadakis IS. Antimicrobial resistance trends among community-acquired respiratory tract pathogens in Greece, 2009-2012. ScientificWorldJournal. 2014; 2014:941564.

25. Khoramrooz SS, Mirsalehian A, Emaneini M, Jabalameli F, Aligholi M, Saedi B, et al. Frequency of Alloicoccus otitidis, Streptococcus pneumoniae, Moraxella catarrhalis and Haemophilus influenzae in children with otitis media with effusion (OME) in Iranian patients. Auris Nasus Larynx. 2012; 39(4):369-73.

26. Hamze M, Osman M, Mallat $H$, Achkar M. Prevalence and antibiotic susceptibility of ear pathogens isolated from patients in Tripoli, north of Lebanon. Int Arab J Antimicrob Agents. 2017; $7(1: 1)$.

27. Christophy R, Osman M, Mallat $H$, Achkar M, Ziedeh A, Moukaddem $W$, et al. Prevalence, antibiotic susceptibility and characterization of antibiotic resistant genes among carbapenem-resistant Gram-negative bacilli and yeast in intestinal flora of cancer patients in North Lebanon. J Infect Public Health. 2017; 10(6):716-20.
28. El Achkar S, Demanche C, Osman $M$, Rafei R, Ismail MB, Yaacoub H, et al. Drug-Resistant Tuberculosis, Lebanon, 2016 2017. Emerg Infect Dis. 2019; 25(3):564-8.

29. El Ashkar S, Osman M, Rafei R, Mallat H, Achkar M, Dabboussi $\mathrm{F}$, et al. Molecular detection of genes responsible for macrolide resistance among Streptococcus pneumoniae isolated in North Lebanon. J Infect Public Health. 2017; 10(6):745-8.

30. Hamze M, Osman M, Achkar M, Mallat H, Dabboussi F. Alarming increase in prevalence of Neisseria gonorrhoeae infections associated with a high level of antibiotic resistance in Tripoli, Lebanon. Int J Antimicrob Agents. 2016; 48(5):576-7.

31. Hamze M, Osman M, Mallat H, Achkar M. Antibiotic Susceptibility of Salmonella spp., Shigella spp. and enteropathogenic Escherichia coli strains isolated from diarrheic children in Tripoli, North Lebanon. Int Arab J Antimicrob Agents. 2016; 6(2:2).

32. Osman M, Al Nasbeh A, Rafei R, Mallat H, Achkar M, Dabbousi $\mathrm{F}$, et al. Characterization of resistance genes to macrolides, lincosamides and streptogramins (MLS) among clinical isolates of Staphylococcus aureus in North Lebanon. Int Arab J Antimicrob Agents. 2015; 5(4:3).

33. Pfaller MA, Farrell DJ, Sader HS, Jones RN. AWARE Ceftaroline Surveillance Program (2008-2010): trends in resistance patterns among Streptococcus pneumoniae, Haemophilus influenzae, and Moraxella catarrhalis in the United States. Clin Infect Dis. 2012; 55 Suppl 3:S187-93.

34. Melo-Cristino J, Fernandes ML, Serrano N, Portuguese Surveillance Group for the Study of Respiratory P. A multicenter study of the antimicrobial susceptibility of Haemophilus influenzae, Streptococcus pneumoniae, and Moraxella catarrhalis isolated from patients with community-acquired lower respiratory tract infections in 1999 in Portugal. Microb Drug Resist. 2001; 7(1):33-8.

\section{Publish in The International}

\section{Arabic Journal of Antimicrobial Agents}

The Journal is an open access peer-reviewed journal that publishes scientific papers about all aspects of antimicrobials. The journal will publish original research articles, reviews, brief reports and case reports dealing with basic and clinical antibacterial agents, antiviral, antiprotozoals, antituberculuous, antifungal and antihelminthes agents. All manuscripts must be prepared in English, and are subject to a rigorous and fair peer-review process. Accepted papers will immediately appear online. The journal aims to advance the knowledge, attitude and the research of chemotherapy in the Arabic world in cooperation with international, national scientific and public societies as well as research centers with similar aims and objectives. 